\title{
Functional MRI evidence for language plasticity in adult epileptic patients: Preliminary results
}

\author{
Emilie Cousin' \\ Monica Baciu' \\ Cédric Pichat' \\ Philippe Kahane ${ }^{2}$ \\ Jean-François Le Bas ${ }^{3}$ \\ 'UMR CNRS/UPMF 5I05, \\ Laboratoire de Psychologie et \\ Neurocognition; ${ }^{2}$ Laboratoire de \\ Neurophysiopathologie de l'Epilepsie, \\ CHU Grenoble; ${ }^{3}$ Unité IRM, CHU \\ Grenoble, France
}

\begin{abstract}
The present fMRI study explores the cerebral reorganisation of language in patients with temporal lobe epilepsy, according to the age of seizures onset (early or late) and the hippocampal sclerosis (associated or not). Seven right-handed control volunteers and seven preoperative adult epileptic patients performed a rhyme decision (language condition) and a visual detection (control condition) tasks in visually presented words and unreadable characters, respectively. All patients were left hemisphere dominant for language. Appropriate statistical analyses provided the following preliminary results: (1) patients compared with healthy subjects showed lower degree of hemispheric lateralization with supplementary involvement of the right hemisphere; (2) the degree of hemispheric specialization depends on the considered region; (3) patients with early seizures show signs of temporal and parietal reorganization more frequently than patients with late onset of seizures; (4) patients with early seizures show a tendency for intra-hemispheric frontal reorganisation; (5) associated hippocampal sclerosis facilitates the inter-hemispheric shift of temporal activation. Although our patients were left hemisphere predominant for language, the statistical analyses indicated that the degree of lateralization was significantly lower than in healthy subjects. This result has been considered as the indication of atypical lateralization of language.
\end{abstract}

Keywords: language, fMRI, plasticity, temporal epilepsy, age, hippocampal sclerosis

\section{Introduction}

The term "focal epilepsy" is classically used for describing seizures generated by a specific cerebral region, the epileptogenic zone (Rosenow and Lüders 2001; Lüders et al 2006). The majority of focal epilepsies in adults are temporal lobe epilepsies (TLE) (Thomas and Genton 1992; Engel 2001), meaning that the region responsible for seizures is situated in the temporal lobe. With respect to healthy subjects, the patients with chronic temporal epileptogenic show reorganization of temporal language networks (Hertz-Pannier et al 2002; Breier et al 2005; Pataraia et al 2004, 2005). As the left temporal lobe is involved in language (Billingsley et al 2001; Seghier et al 2004; Gitelman et al 2005), an atypical representation (bilateral hemispheric representation or right hemispheric predominance) of the subjacent cerebral regions, could occur (Adcock et al 2003; Demonet et al 2005). Overall, when the epileptogenic zone is located within the predominant hemisphere for language, two types of cerebral reorganization have been generally described: (a) intra-hemispheric, ie, the nonaffected cerebral regions from the hemisphere where the epileptogenic zone is located in, take over language functions, for instance, the language function of the temporal region could "shift" within the frontal region of the same hemisphere, when the epileptogenic zone is situated in the temporal lobe (Bell et al 2002; Brazdil et al 2003; Pataraia et al 2004, 2005; Kadis et al 2007) and (b) inter-hemispheric, ie, the homologues cerebral regions situated within the nonpredominant hemisphere for language become involved in language (Hertz-Pannier et al 2002; Pataraia et al 2004, 2005; Breier et al 2005; 
Thivard et al 2005; Helmstaedter et al 2006). The type of reorganization may depend on many factors such as the age of seizures onset (Hertz-Pannier et al 2002; Liegeois et al 2004; Berl et al 2005; Demonet et al 2005; Goldmann and Golby 2005; Thivard et al 2005), the considered language region, temporo-parietal or frontal (Kurthen et al 1992; Hertz-Pannier et al 2002; Liegeois et al 2004; Thivard et al 2005) or the associated hippocampal sclerosis (Knecht 2004; Janszky et al 2006; Weber et al 2006).

The factor which has been often studied within this framework, was the age of seizures onset (Muller et al 1999a; Bell et al 2002; Brazdil et al 2003; Pataraia et al 2004). The onset of seizures before age of six (Goldmann and Golby 2005; Yuan et al 2006) and originated in the left predominant hemisphere could induce either inter-hemispheric (bilateral or right hemispheric) (Liegeois et al 2004; Muller et al 1999a, 1999b; Voets et al 2006) or intra-hemispheric (Bell et al 2002; Pataraia et al 2004) reorganization of language. Overall, it is generally accepted that the right shift of language tends to be stronger and concerns more numerous language regions in patients with early seizures, as compared to patients with late (after age of six) lesions (Muller et al 1999a). The intrahemispheric reorganization means that language operations depend on regions which are not originally dedicated to these operations. Thus, the incidence of the intra-hemispheric reorganization is significantly higher if seizures start early in life (Bell et al 2002; Pataraia et al 2004).

Epileptics with associated hippocampal sclerosis are more likely to show atypical reorganisation of language than patients without sclerosis (Janszky et al 2003; Knecht 2004; Liegeois et al 2004; Weber et al 2006; Maccotta et al 2007). It has been suggested that hippocampal pathology influences the intra- and the inter-hemispheric reorganization of language (Knecht 2004). By using fMRI, it has been shown that patients with left hippocampal sclerosis show high involvement of the right medial temporal lobe during synonym judgement (Weber et al 2006) or living categorization (Richardson et al 2003). During performing a language task, epileptics with hippocampal sclerosis show evidence for supplementary ipsilateral recruitement of nontemporal areas such as frontal regions (Maccotta et al 2007). These studies suggest inter-hemispheric temporal as well as intrahemispheric reorganisation of language when the hippocampal sclerosis is associated.

Also, the pattern of language plasticity depends on the language region which is considered. Within this framework, Thivard and colleagues (2005) have shown that the inter-hemispheric shift of language from the left to the right hemisphere is more likely to occur for temporal than for frontal regions in patients with TLE. Indeed, they showed that temporal lateralization indices indicate supplementary involvement of the right hemisphere, than frontal lateralization indices. These findings are in agreement with results provided by Billingsley and colleagues (2001) showing larger inter-hemispheric shift of the activation for temporal than for frontal regions. These results suggest that language plasticity should be investigated at regional rather than at global hemispheric level.

The aim of this fMRI study was to assess the possible reorganization of language regions in epileptic patients with focal temporal lobe epilepsy when they are compared to healthy participants. The language reorganisation was explored at regional (frontal, temporal, parietal) level and the influence of two factors, the age of seizures onset and the associated hippocampal sclerosis, has been explored. All subjects performed a rhyme detection task. This task induces robust lateralized activation within the predominant hemisphere for language (Lurito et al 2000; Baciu et al 2001; Billingsley et al 2001; Seghier et al 2004; Baciu, Watson et al 2005; Cousin et al 2006) and particularly along the left perisylvian (frontal, temporal, parietal) regions (Pugh et al 1996; Baciu, Juphard et al 2005) essential for language (Ojemann et al 1989). Thus, by using this task we could explore the possible reorganisation at global hemispheric level as well as at regional level.

Our hypotheses were the following:

- At hemispheric level, the difference between left and right hemisphere activity should be greater in healthy participants than in epileptic patients due to significant involvement of the right hemisphere,

- At regional level, the reorganisation would significantly concern temporo-parietal than frontal regions, as the epileptogenic zone is temporal,

- The TLE associated with hippocampal sclerosis would induce higher degree of atypical temporal lateralization than TLE without hippocampal sclerosis.

\section{Material and methods}

\section{Participants}

The explored population included seven healthy right-handed (Oldfield 1971), native French speaker volunteers (3 women and 4 men, mean of age 28.28 years old) with no history of neurological disorders and seven preoperative adult patients (see Table 1 for demographic data) with medically intractable TLE. Among them, 6/7 had left TLE, 4/6 had ipsilateral hippocampal sclerosis, $4 / 7$ had early seizures (before age of six; 
Table I Shows demographic data for the epileptic patients examined in this study. For each of them the following information is mentioned

\begin{tabular}{|c|c|c|c|c|c|c|c|c|c|c|c|}
\hline Patients & $\begin{array}{l}\text { Age, } \\
\text { Sex }\end{array}$ & Handedness & VIQ & $\begin{array}{l}\text { Age at } \\
\text { seizures } \\
\text { onset }(y)\end{array}$ & VEEG & SEEG & EZ location & $\begin{array}{l}\text { HD } \\
\text { VEEG }\end{array}$ & $\begin{array}{l}\text { HD } \\
\text { SEEG }\end{array}$ & $\begin{array}{l}\text { HD } \\
\text { fmRI }\end{array}$ & $\begin{array}{l}\text { Hippocampal } \\
\text { sclerosis }\end{array}$ \\
\hline I. & $14, \mathrm{~m}$ & $R$ & 105 & 12 & $\begin{array}{l}\text { Clinical features } \\
\text { and EEG spikes }\end{array}$ & Yes & $\begin{array}{l}\text { L temporal } \\
\text { (VEEG, SEEG) }\end{array}$ & - & $\mathrm{LH}$ & $\mathrm{LH}$ & - \\
\hline 2. & $15, \mathrm{~m}$ & $\mathrm{R}$ & $\begin{array}{l}\text { Not } \\
\text { available }\end{array}$ & 12 & $\begin{array}{l}\text { Intercritical } \\
\text { EEG spikes }\end{array}$ & Yes & $\begin{array}{l}\text { L temporal } \\
\text { (VEEG, SEEG) }\end{array}$ & LH & LH & $\mathrm{LH}$ & - \\
\hline 3. & $39, f$ & L & 95 & 14 & $\begin{array}{l}\text { Clinical features } \\
\text { and EEG spikes }\end{array}$ & Yes & $\begin{array}{l}\text { R temporal } \\
\text { (VEEG, SEEG) }\end{array}$ & $\mathrm{LH}$ & $\mathrm{LH}$ & $\mathrm{LH}$ & $R$ \\
\hline 4. & $17, f$ & L & $\begin{array}{l}\text { Not } \\
\text { available }\end{array}$ & 3 & Clinical features & Yes & $\begin{array}{l}\text { L temporal } \\
\text { (VEEG, SEEG) }\end{array}$ & - & $\mathrm{LH}$ & $\mathrm{LH}$ & - \\
\hline 5. & $17, f$ & $\mathrm{R}$ & 85 & 5 & EEG spikes & Yes & $\begin{array}{l}\text { L temporal } \\
\text { (VEEG, SEEG) }\end{array}$ & $\mathrm{LH}$ & LH & LH & L \\
\hline 6. & $43, m$ & L & 100 & 1 & $\begin{array}{l}\text { Clinical features } \\
\text { and EEG spikes }\end{array}$ & No & $\begin{array}{l}\text { L temporal } \\
\text { (VEEG) }\end{array}$ & LH & - & $\mathrm{LH}$ & L \\
\hline 7. & $34, f$ & $\mathrm{R}$ & $\begin{array}{l}\text { Not } \\
\text { available }\end{array}$ & 5 & EEG spikes & No & $\begin{array}{l}\text { L temporal } \\
\text { (VEEG) }\end{array}$ & $\mathrm{LH}$ & - & $\mathrm{LH}$ & L \\
\hline
\end{tabular}

Age; Sex; Handedness ( $\mathrm{R}$ = right-handed; L = left-handed), VIQ (verbal IQ assessed by Mill Hill Part B or WAIS III), age of seizures onset, VEEG (video-EEG monitoring showing the clinical features and the EEG during seizures or intercritical); SEEG (stereo-EEG); Location of the EZ (epileptogenic zone); HD = hemispheric predominance assessed by VEEG, SEEG, fMRI (all patients had LH = left hemispheric predominance);Associated hippocampal sclerosis ( $\mathrm{L}=$ left or $\mathrm{R}=$ right). In all patients the neurological examination was normal and MRI excluded tumours or scars. The epileptogenic zone in all patients was dysplasia.

mean of age 3.5) and 3/7 had late seizures (after age of six; mean of age 12.3). Although 4/7 patients were right-handed and 3/7 were left-handed, they were considered together in the analyses. Each patient included in this study was diagnosed in the neurology department and the type of seizures was determined based on the clinical features, video-EEG and stereoEEG. None patient underwent surgery at the time of the present study. The temporal location of the epileptogenic zone was determined by VEEG and by using SEEG (5/2 patients). All patients had "functional" epileptogenic zone, meaning that no tumours or scars was detected to MRI, although some of them had hippocampal sclerosis. In all patients the left hemisphere was predominant for language (as determined by VEEG and SEEG and confirmed by fMRI examination, see Table 1). This study has been reviewed by the appropriate ethics committee and all participants gave their informed consent.

\section{Stimuli}

The stimuli consisted of couples of words presented visually one above the other in the middle of the screen. The words were written either in a readable (Times) or in an unreadable (Karalyn Patterson) font. Overall, 58 couples of readable and 58 couples of unreadable words were presented per scan. The stimuli were generated by means of Psyscope V.1.1 (Carnegie Mellon Department of Psychology) on a Macintosh computer (Power Macintosh 9600). They were transmitted into the magnet by means of a video projector (Eiki LC
6000), a projection screen situated behind the magnet and a mirror centred above the patient's eyes. The duration of each stimulus (couple of readable and unreadable words) was $3 \mathrm{sec}$. Examples of stimuli presented during the experiment are shown in Figure 1.

\section{Tasks}

Patients and control subjects were instructed to judge whether the words rhymed (rhyme detection task, "task epoch"). With respect to "words" in unreadable font, patients and controls were instructed to judge if "words" contained at least one character which overshot the others (visual detection task, "control epoch"). The "yes" and "no" responses for the two epochs were transmitted by means of two keys pressed with the index and the middle finger of the dominant hand, respectively.

\section{Paradigm and MR acquisition}

A "block" paradigm was used during the fMRI examination, consisting of five periods, each composed of a "control" and a "task" epochs. Each epoch lasted 48 seconds. Each patient underwent a single fMRI scan. Before entering into the magnet, the subjects were positioned in front of a computer screen, and carefully instructed and trained to execute the tasks by using stimuli which were different from those used during the experiment.

Functional MR imaging was performed on a 1.5 Tesla MR imager (Philips NT) equipped with echo-planar (EPI) 


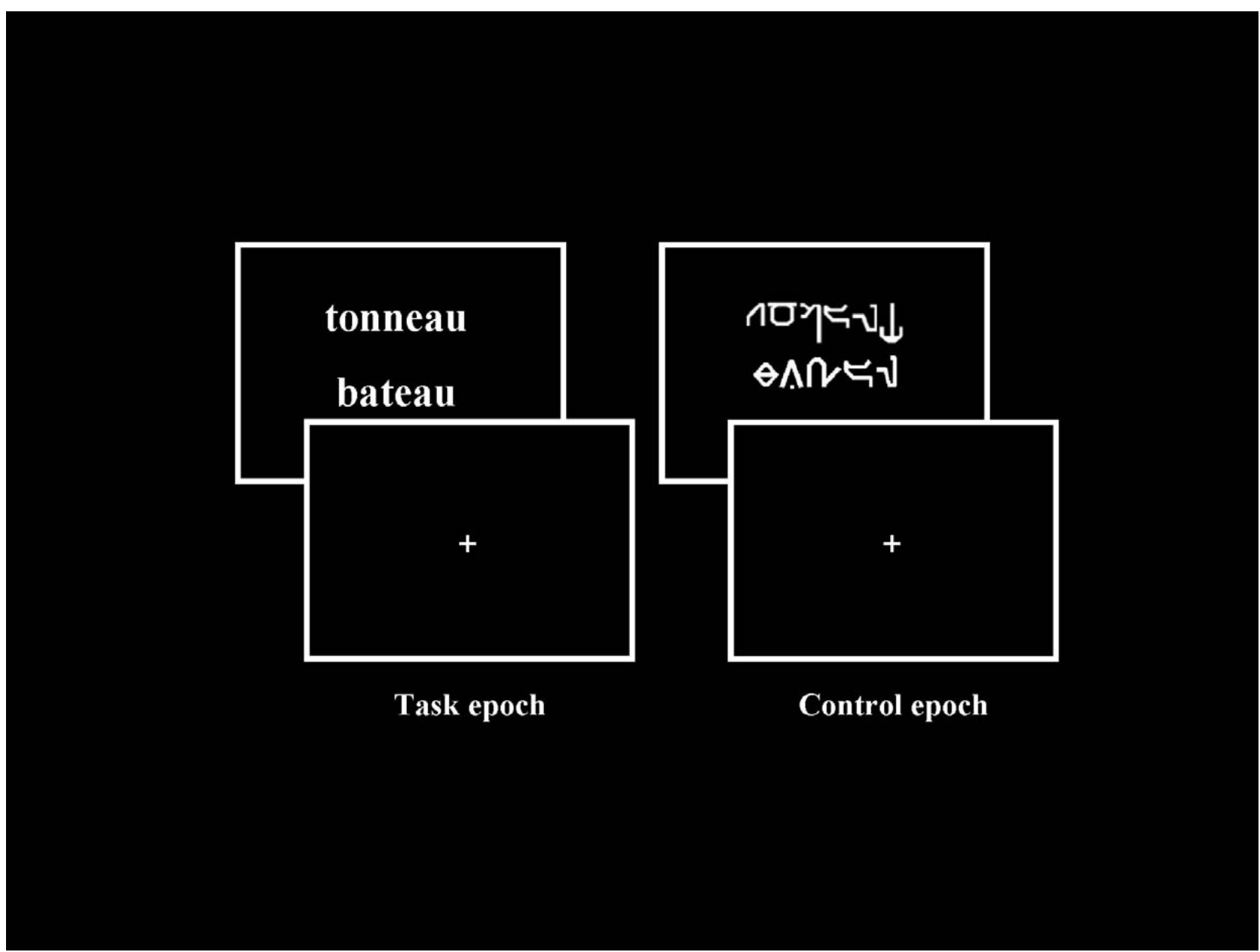

Figure I Examples of stimuli presented during fMRI paradigm, respectively "Task" (pairs of readable words) and "Control” (pairs of unreadable words written in Karalyn Patterson font) conditions. Concerning the "Task", patients and controls were instructed to judge whether the words rhymed (rhyme detection task, "task epoch"). Concerning the "Control", patients and healthy controls were instructed to judge whether the words contained at least one character which overshot the others.

acquisition. Twenty five adjacent, axial slices (thickness $5 \mathrm{~mm}$ each) were imaged sequentially. The imaging volume was oriented parallel to the bicommissural (AC-PC) plane. It was measured sixteen times during each epoch. Positioning of the image planes was performed on scout images acquired in the sagittal plane. An EPI MR Pulse sequence was used. The major MR acquisition parameters of this sequence were: $T R=3000 \mathrm{~ms}$, $\mathrm{TE}=45 \mathrm{~ms}$, flip angle $=90^{\circ}$, acquisition time per slice $=37 \mathrm{~ms}$, field-of-view $=256 \times 256 \mathrm{~mm}^{2}$, imaging matrix $=64 \times 64$, reconstruction matrix $=128 \times 128$. Subsequent to the functional scan, a high resolution $3 \mathrm{D}$ anatomical MR scan was obtained from the volume examined functionally.

\section{Data processing}

Data analysis was performed by using the SPM2 software (Friston et al 1995) and based on the general linear model (boxcar function convolved with the hemodynamic response). The MR images were spatially pre-processed as follows. First, motion correction was applied and all images were realigned by means of a rigid body transformation with respect to the first acquired image. Subsequently, all images (patients and controls) were normalized, first the anatomical and then, the functional volumes. In order to avoid left-right morphological asymmetries that could bias the inter-hemispheric comparison, a symmetrical template was built by averaging the standard MNI template and its mirror with respect to the mid-sagittal plane. This average template was then used during for normalization of all participants including patients, as they did not have anatomical brain deformation, confirming by MRI. To conform to the underlying assumption in SPM that the data are normally distributed, the functional images were spatially smoothed by an 8 mm FWHM (Full Width at Half Maximum) Gaussian kernel. Time-series for each voxel were high-pass filtered $(1 / 128 \mathrm{~Hz}$ cutoff) to remove low-frequency noise and signal drift.

The general linear model was then used to generate parameter estimates of activity at each voxel, for each 
condition, and each participant. Statistical parametric maps were generated from linear contrasts between the HRF parameter estimates for the two experimental conditions. At the individual level, we assessed the whole network of cerebral areas involved for each contrast (rhyme vs control; control vs rhyme). Subsequently, we performed in healthy participants a random-effect group analysis on the contrast images from individual analyses (Friston et al 1998), using one-sample $t$ tests. Clusters of activated voxels were then identified as significant at a voxel level of $\mathrm{p}\langle 0.001, \mathrm{~T}\rangle$ 4.50 , extended threshold of 15 voxels. The group analysis allowed identifying language regions significantly more activated within one hemisphere with respect to the other one. The brain regions were reported according to the stereotaxic atlas of Talairach and Tournoux (1988) and the activation's peak ( $x, y, z$ coordinates) of regions of interest (ROI) have been identified. The considered ROIs were the regions classically activated by previous studies for this task for healthy participants (Demonet et al 1992; Lurito et al 2000; Baciu et al 2001; Seghier et al 2004; Baciu, Juphard et al 2005). They were the inferior frontal gyrus (IFG, BA 44, 45), the inferior and middle temporal regions (IMTG, BA 37, 21) and the inferior parietal lobule (IPL, BA 39, 40). Subsequently, the peak of activation was used for drawing individual ROI in each participant (healthy controls and patients) by using the Marsbar tool (see http://marsbar.sourceforge.net). Each ROI was delineated as a sphere of $20 \mathrm{~mm}$ radius (Liegeois et al 2004) centred on the peak $(x, y, z)$ and symmetrically within hemispheres (one ROI to the left and the symmetrical to the right for each participant). Then, the parameter estimates ( $\%$ of signal intensity variation for task and control conditions) were extracted from all left and right ROIs, in each participant.

\section{Statistical analysis of the parameter estimates in patients}

The parameter estimates values for all left ROIs and all right ROIs of individual participants were submitted to a three way ANOVA analysis (by using STATISTICA 5.0 software) with activated regions (IFG, IMTG, IPL) and hemispheres (Right and Left) as within-subjects factors, and pathological condition (Controls and Patients) as between-subject factor. We checked first if patients were significantly differently lateralized than healthy subjects. If significant difference in lateralization between the groups has been detected, we subsequently tested for each ROI if the factors of interest (age of seizures onset and associated hippocampal sclerosis) influenced plasticity. Their influence was checked for the intra- and inter-hemispheric reorganization of language. The parameter estimates for each left and the homologue right ROI were correlated with the two variables (age of onset and associated hippocampal sclerosis). Because of the small sample size, a nonparametric procedure was used (Spearman correlations). The critical significance threshold of correlations was considered at $p<0.05$. Significant correlations suggested that these factors have an effect on the cerebral reorganization of language in epileptic patients.

\section{Results}

\section{Healthy participants}

Activated regions (ROI) during rhyming task

Brain areas involved in "task" (rhyme) condition relative to "control" (visual detection) and in "control" relative to "task" are summarized in Table 2. During rhyme, the activated regions of interest were exclusively located to the left: inferior frontal gyrus (IFG, BA 44, $x y z,-40$ 1725), inferior parietal lobule (supramarginal gyrus, SMG, BA 40, $x y z,-44-4135$ ) and infero-medial temporal gyrus (IMTG, BA 37/21, xy z, -44-63-7). During visual detection, the activated regions were exclusively located to the right: middle frontal gyrus (BA 9, $x y z, 511036)$ and middle occipital gyrus (BA 19, $x y z, 44-40-15$ ).

\section{Epileptic patients}

MR signal intensity variation (parameter estimates, magnitude) in left and right ROI in individual patients The parameter estimates were extracted from the ROI identified in healthy subjects: IFG, IPL (SMG), and IMTG. Then, these values were subsequently analysed by using ANOVA analyses. First, when all participants (Controls and Patients) and all regions (IFG, IPL, IMTG) were considered, we obtained significantly greater activation of the left $\left(F_{1,12}=\right.$ $5.295, \mathrm{p}<0.05)$ than of the right hemisphere. Furthermore, ANOVA revealed an interaction (Figure 2) between hemispheres and pathological condition; $\left(F_{1,12}=7.60, \mathrm{p}<0.01\right)$ with greater inter-hemispheric difference (ie, left hemispheric dominance) for healthy than for epileptic patients. This result suggests that right hemisphere is supplementary involved in patients than in controls during language, although patients keep left hemispheric predominance.

\section{The effect of seizures onset and hippocampal sclerosis on plasticity}

This effect was assessed for each individual left ROI and right ROI by performing correlations between the parameter estimates extracted from these regions and the two variables (age of seizures onset and associated hippocampal sclerosis). By using the WFUpickatlas (Maldjian et al 
Table 2 Cerebral activated regions obtained for "Task" versus "Control" (T $>C$ ) and for "Control" versus "Task" (C $>$ T). The statistical significance threshold for individual voxels was set at $\mathrm{p}<0.00 \mathrm{I}$ uncorrected (random-effect analysis). The Talairach peak coordinates $(x, y, z)$ are indicated for each cluster

\begin{tabular}{|c|c|c|c|c|c|c|c|c|}
\hline Contrast & Cerebral activated regions & $\mathbf{H}$ & BA & k & $x$ & $y$ & $\mathbf{z}$ & $T$ \\
\hline \multirow[t]{9}{*}{ T vs $C$} & Frontal cortex & & & & & & & \\
\hline & Inferior frontal gyrus & L & 44 & 133 & -40 & 17 & 25 & 10.75 \\
\hline & Superior frontal gyrus & $\mathrm{R}$ & II & 22 & 32 & -63 & -24 & 5.79 \\
\hline & Parietal cortex & & & & & & & \\
\hline & Inferior parietal lobule & & & & & & & \\
\hline & (supramarginal gurus) & L & 40 & 114 & -44 & -41 & 35 & 8.52 \\
\hline & Temporal cortex & & & & & & & \\
\hline & Inferomedial temporal gyrus & L & $21 / 37$ & 41 & -44 & -63 & -7 & 5.95 \\
\hline & Cerebellum & L & - & 46 & -16 & -90 & -6 & 6.77 \\
\hline \multirow[t]{5}{*}{ C vs T } & Frontal cortex & & & & & & & \\
\hline & Middle frontal gyrus & $\mathrm{R}$ & 9 & 22 & 51 & 10 & 36 & 5.65 \\
\hline & Occipital cortex & & & & & & & \\
\hline & Middle occipital gyrus & $\mathrm{R}$ & 19 & 55 & 44 & -40 & -15 & 7.27 \\
\hline & Cerebellum & L & - & 46 & -16 & -90 & -6 & 6.77 \\
\hline
\end{tabular}

Abbreviations: $\mathrm{H}$, hemisphere; $\mathrm{R}$, right hemisphere; L, left hemisphere; BA, Brodmann area; $k$, number of voxels in the cluster; T, rhyme task; $\mathrm{C}$, control task.

2003, 2004) we illustrate in Figures 3-5 only cerebral regions suggesting reorganization ("plastic" regions) with respect to control subjects, according to the age of seizure onset (IFG, IMTG, IPL) and the associated hippocampal sclerosis (IMTG). In the following section we describe each specific region.
Inferomedial temporal gyrus (IMTG, BA 37/2I)

We obtained significant correlations between the parameter estimates extracted from the left IMTG and the age of seizures onset $\left(\mathrm{r}_{\mathrm{s}}=0.83 ; \mathrm{p}<0.01\right)$, as well as between the parameters estimates extracted from the right IMTG and the age of seizures onset $\left(r_{s}=0.89 ; p<0.006\right)$. This result suggests

\section{Signal\%}

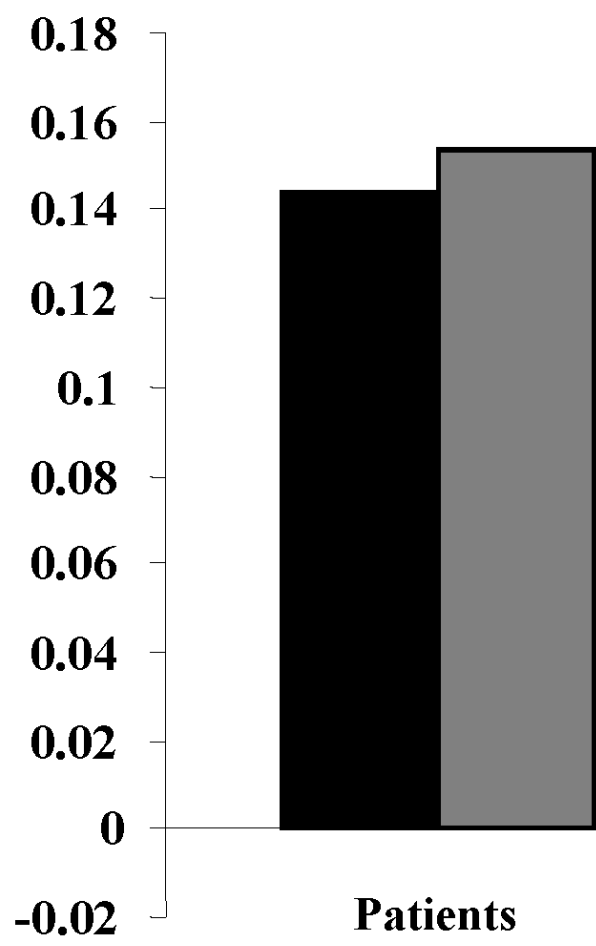

- Left hemisphere

$\square$ Right hemisphere $\dot{*}$

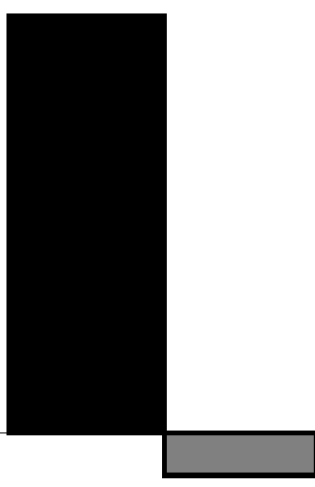

Control subjects

Figure 2 Significant interaction between hemisphere (Left and Right) and pathological condition (Control and Patient). The y-axis shows the degree of hemispheric lateralization (magnitude difference between conditions in left and right ROI for patients and controls ( $x$-axis). It shows significantly left-right difference for controls with respect to patients. 
that patients presenting seizures early in life have more bilateral activation of the IMTG (Figure 3a). Interestingly, we also obtained significant correlation between the parameters estimates extracted from the right IMTG and the associated left hippocampal sclerosis $\left(r_{s}=0.77 ; p<0.05\right)$. This result suggests that patients with associated hippocampal sclerosis are more likely to involve their homologue IMTG during language (Figure 3b).

Inferior parietal lobule (IPL, SMG, BA 40)

Significant correlation was obtained between the parameters estimate extracted from the right IPL and the age of seizures onset $\left(r_{s}=0.89 ; p<0.006\right)$. This result suggests that early age of seizures onset could induce supplementary activation of the right homologue parietal region in patients (Figure 4).

No significant correlation has been obtained between the parameter estimates extracted from this region and the presence/absence of hippocampal sclerosis.

\section{Inferior frontal gyrus (IFG, BA 44)}

The correlation between the parameter estimates extracted from the left IFG and the age of seizures onset failed to reach significance $\left(r_{s}=0.71 ; p=0.06\right)$ suggesting only a tendency of the intra-hemispheric reorganisation for this region when seizures start early in life (Figure 5).

No significant correlation was observed between the parameter estimates extracted from the IFG and the presence/absence of hippocampal sclerosis.

Our patient population was not homogeneous with respect to the handedness: 4/7 of patients were righthanded and 3/7 were left-handed. This sample was too small for splitting patients in right and left-handed. Although the handedness was confounded because we did not explore the relationship between handedness and cerebral reorganisation, we checked the possible effect of this factor. On this purpose, Spearman correlations between handedness and left and right homologues ROI have been calculated. None significant effect was obtained $\left(0.43<\mathrm{r}_{\mathrm{s}}<0.14 ; 0.33<\mathrm{p}<0.75\right)$ suggesting that the handedness did not significantly affect the cerebral reorganisation in our sample.

\section{Discussion}

This fMRI study explored the influence of two variables (age of seizures onset and associated hippocampal sclerosis) on the cerebral reorganisation of language in seven adult epileptic patients with respect to seven healthy subjects, while they performed a rhyme detection task. We first identified language ROI based on a randomeffect group analysis for delineating their own left and symmetrical right ROI. From each ROI we extracted the parameters estimates (magnitude or \% of MR signal variation) for each condition ("task" and "control"). We included these values in ANOVAs for detecting significant differences between patients and controls within these ROI. In patients, we also estimated (Spearman correlations) the effect of the two variables (age of seizures onset and hippocampal sclerosis) on the reorganization of language.

\section{Regions of interest during rhyme detection}

This task presupposes predominant grapho-phonemic conversion (phonological analysis) of words in order to detect rhyming but also automatic semantic analysis when a word is presented (Baciu et al 2001; Seghier et al 2004). That is why this task was used in the present study for assessing regions related to "language" (both, phonological and semantic aspects of language). The task induced activation of regions predominantly located in the left hemisphere, confirming results obtained by previous studies (Demonet et al 1992; Binder et al 1996, 1997; Lurito et al 2000; Price 2000). We obtained (a) left frontal activation (BA 44, $x y z,-401725$ ) responsible for output phonology (Demonet et al 1992), planning and execution of language (ie, articulation) and post-lexical processing (Gitelman et al 2005), (b) left temporal activation (BA $37 / 21, x$ y $z,-44-63-7)$ related to semantic associations (Lurito et al 2000; Billingsley et al 2001; Gitelman et al 2005; Schaefer et al 2006) and (c) left parietal activation (BA 40, $x$ y $z,-44-4135$ ) related to short term memory phonological storage (Gitelman et al 2005). We briefly remind that visual detection ("control") induced very limited activation within the right hemisphere, related to visual attentional processes particularly involved for performing this task (Corbetta et al 1998).

\section{Hemispheric lateralization of language in patients with respect to control subjects}

Our results show that patients have significantly reduced left hemispheric lateralization for language than righthanded healthy participants who are classically strongly left lateralized (Fiez 2001; Seghier et al 2004; Baciu, Juphard et al 2005). The reduced left hemisphere predominance suggests a tendency for bilateralization with supplementary 


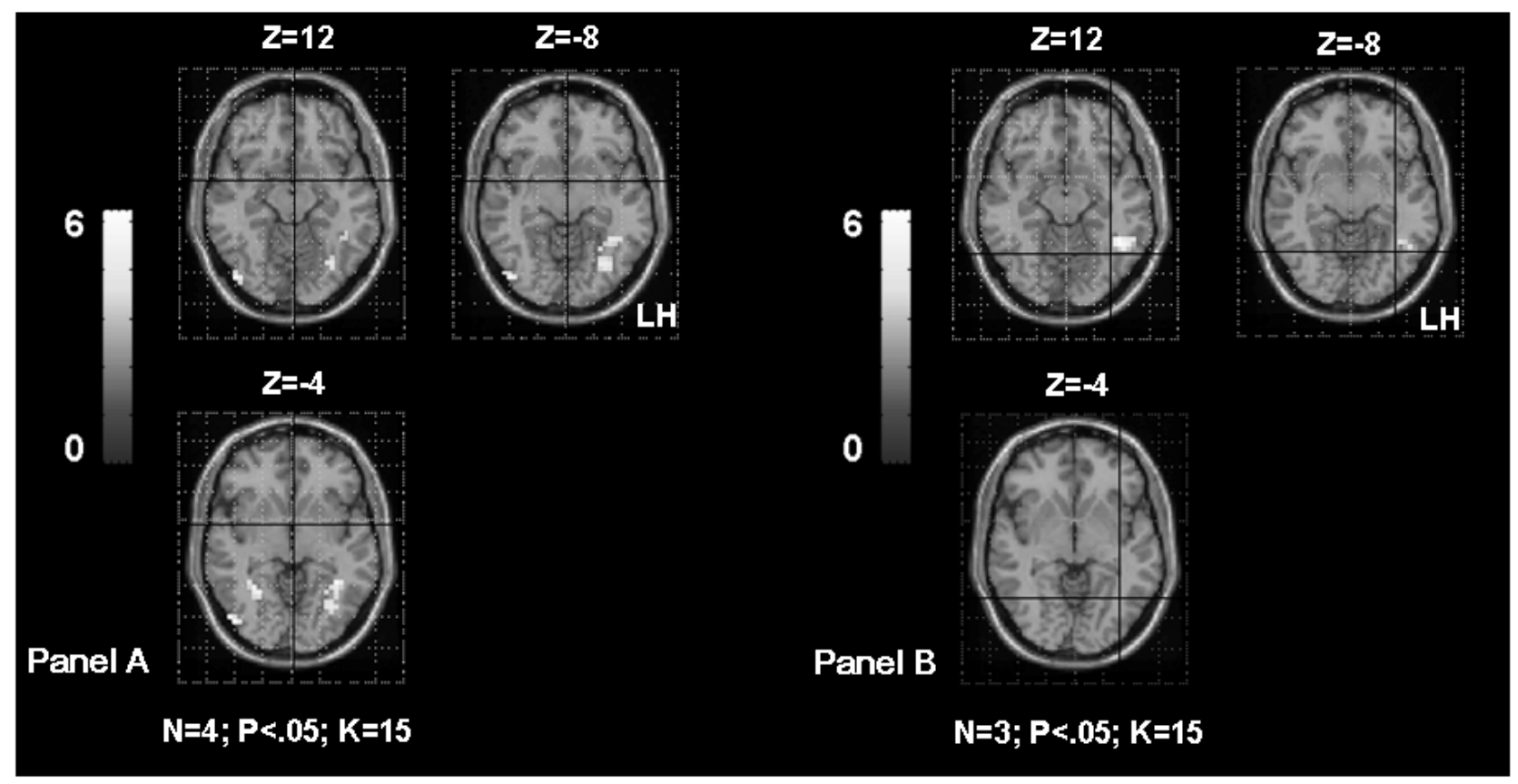

Figure 3a Shows the inferomedial temporal gyrus (IMTG) activation in TLE patients according to the age of seizures onset. The Panel A shows bilateral IMTG activation in patients with seizures starting before age of 6 years old (OC, SDi, PD, CG, Table I). The Panel B shows left IMTG activation in patients with seizures starting after age of 6 years old (MR, FD, SD, Table I). The activation is projected onto 2D anatomical slices (left is right).

involvement of the right hemisphere (Springer et al 1999; Adcock et al 2003). Other studies have previously shown change of the hemispheric specialization degree or pattern in patients (Pataraia et al 2004, 2005; Brazdil et al 2005;
Thivard et al 2005; Yuan et al 2006) with respect to healthy subjects. However, in these studies, the assessment of the left-right hemispheric difference was not statistically robust as they generally used lateralization indices calculation.

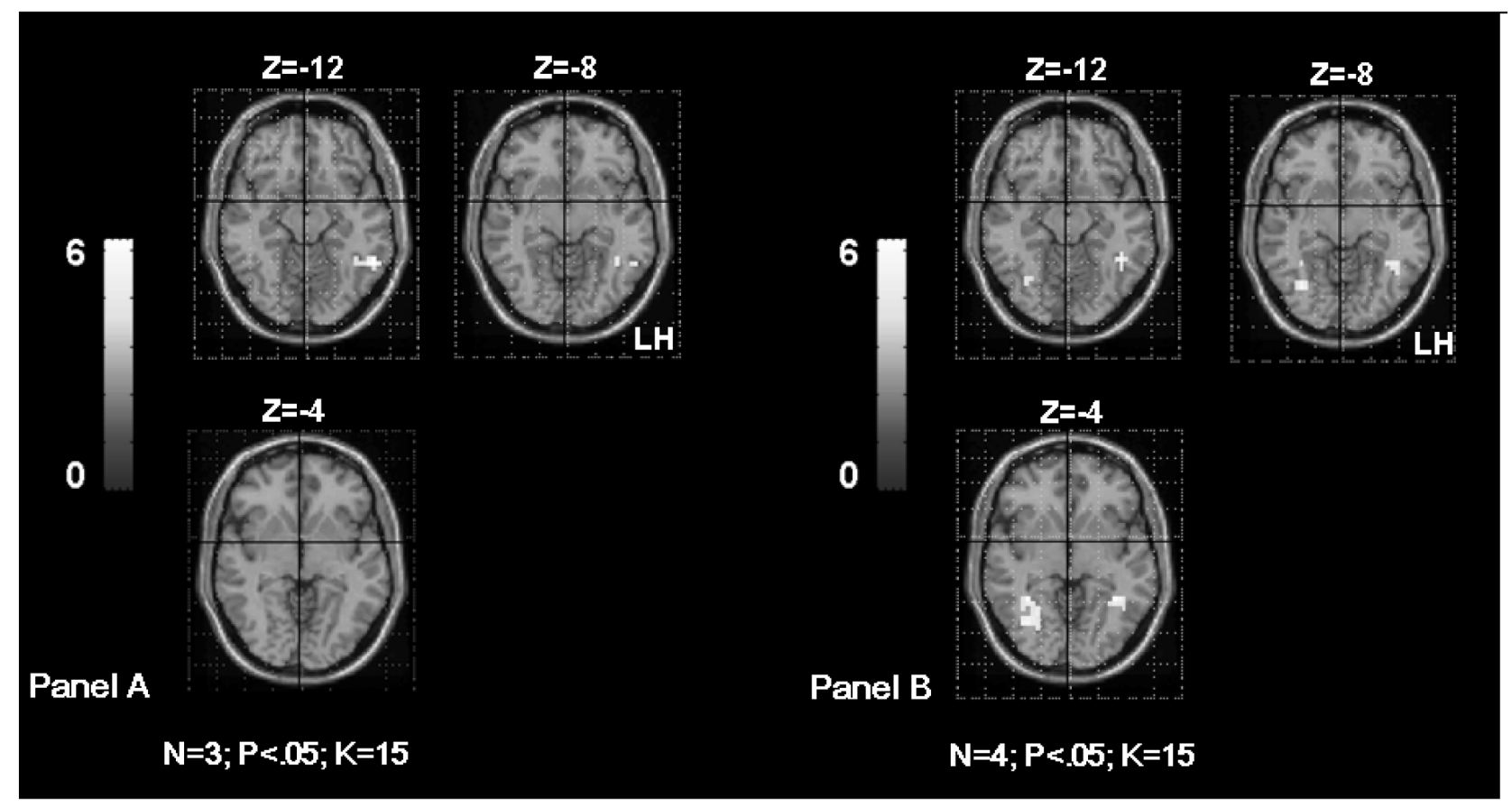

Figure 3b Shows the inferomedial temporal gyrus (IMTG) activation in TLE patients according to the associated hippocampal sclerosis. The Panel A shows left IMTG activation in patients without hippocampal sclerosis (MR, FD, OC from Table I). The Panel B shows bilateral IMTG activation in patients with hippocampal sclerosis (SD, $\mathrm{SDi}, \mathrm{PD}, \mathrm{CG}$ from Table I). The activation is projected onto 2D anatomical slices (left is right). 


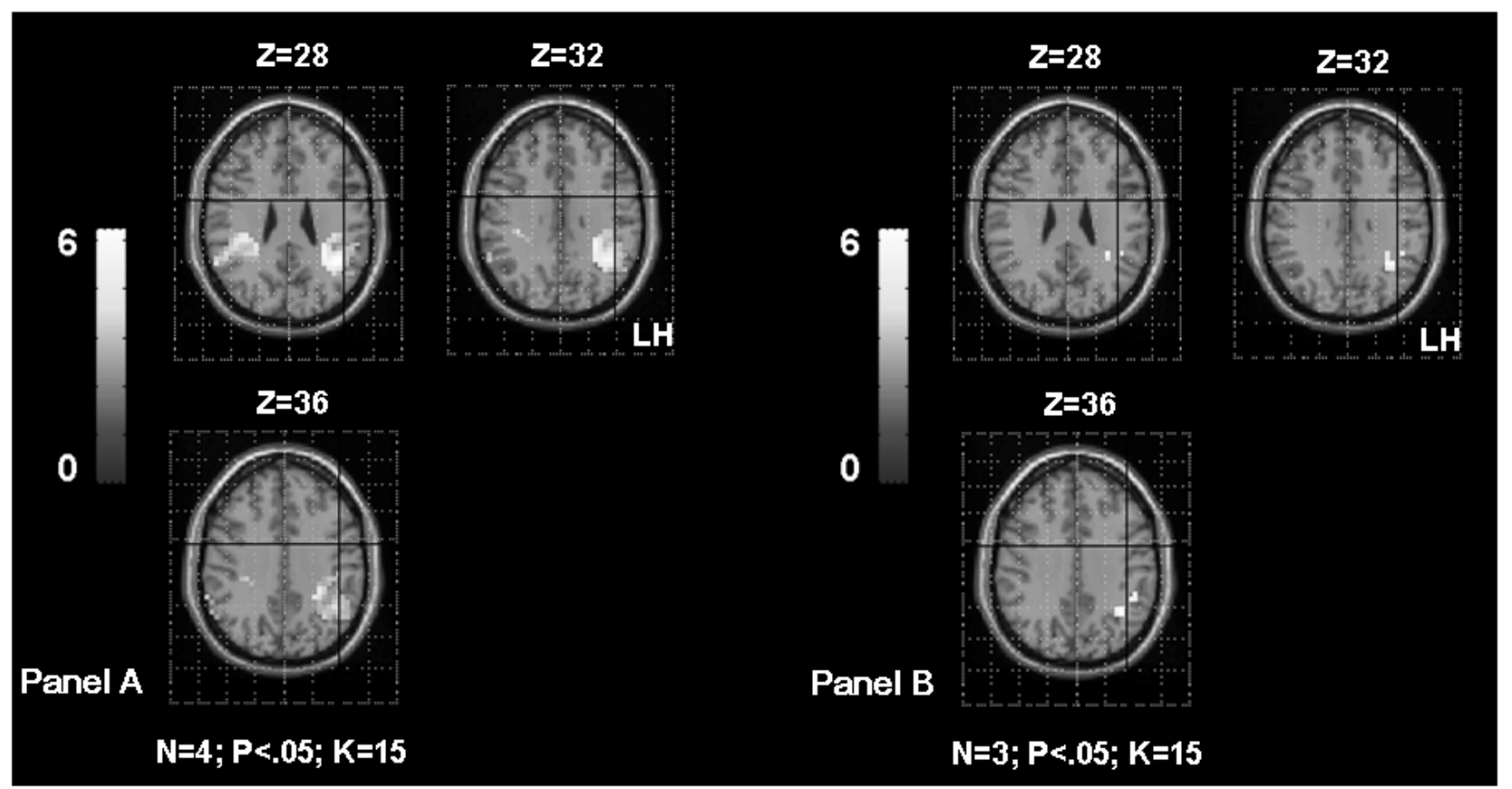

Figure 4 Shows the supramarginal gyrus (SMG) activation in TLE patients according to the age of seizures onset. The Panel A shows bilateral SMG activation in patients with seizures starting before age of 6 years old (OC, SDi, PD, CG from Table I). The Panel B shows left SMG activation in patients with seizures starting after age of 6 years old (MR, FD, SD from Table I). The activation is projected onto 2D anatomical slices (left is right).

This method is known as being threshold-dependent and based on the number of activated voxels within hemispheres (Liegeois et al 2002; Baciu, Juphard et al 2005). In the present study, we brought robust evidence suggesting differences in the lateralization pattern in patients and in controls, by using robust statistical analyses on the MR signal intensity variation (parameter estimates) extracted from ROIs.

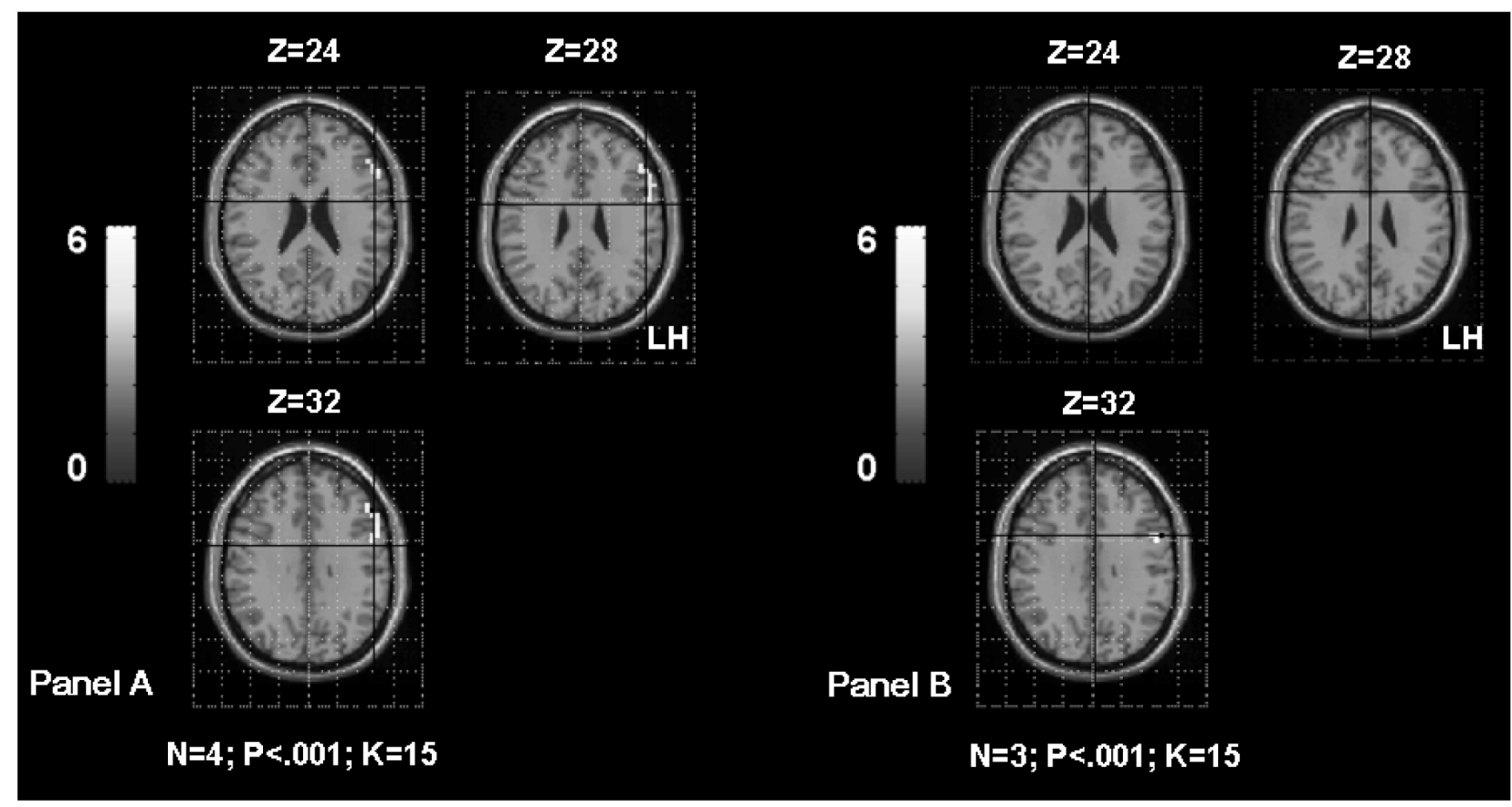

Figure $\mathbf{5}$ Shows the inferior frontal gyrus (IFG) activation in TLE patients according to the age of seizures onset. The Panel A shows left IFG activation in patients with seizures starting before age of 6 years old (OC, SDi, PD, CG from Table I). The Panel B shows left IFG activation in patients with seizures starting after age of 6 years old (MR, FD, SD from Table I). The first sub-group had significantly higher activation of the left frontal region than the second sub-group.The activation is projected onto $2 \mathrm{D}$ anatomical slices (left is right). 


\section{The effect of seizures onset and associated hippocampal sclerosis on language reorganisation}

As suggested by other studies (Lehericy et al 2002; Ries et al 2004; Thivard et al 2005), the language reorganization should be explored at regional rather than at hemispheric level, because the reorganisation pattern depend on the region taken into account. Furthermore, the reorganisation of temporal regions occurs more frequently than the reorganisation of frontal regions. Several factors can influence the reorganisation pattern. In this study we explored two of them:

\section{Age of seizures onset}

The age of seizures influences cerebral plasticity (HertzPannier et al 2002; Brazdil et al 2003; Liegeois et al 2004; Thivard et al 2005). Althought it is difficult to distinguish between neuropathological changes and normal language development (Yuan et al 2006), it is largely accepted that the age is the primary factor for predicting recovery with prognosis so far better in children under age of six. Thus, under the age of six, the language plasticity is reflected by supplementary involvement of the homologue hemisphere and decrease of the degree of specialization (Muller et al 1999a; Brazdil et al 2003; Liegeois et al 2004; Demonet et al 2005; Pataraia et al 2005; Thivard et al 2005; Helmstaedter et al 2006; Voets et al 2006; Yuan et al 2006). Is is also reflected by intra-hemispheric reorganization of cerebral networks (Lazar et al 1997; Bell et al 2002; Pataraia et al 2004), meaning that regions not originally involved in a specific language operation, become involved after reorganization. In the present study, the age of seizures onset was significantly correlated with the activity of each ROI taken into account. Next, we describe how the cerebral plasticity could occur in each of the "plastic" regions.

\section{Frontal region}

Our results suggest only a tendency of an intra-hemispheric reorganization of frontal activation with respect to healthy subjects. This tendency suggests that early seizures induce greater activation/involvement of frontal region than seizures starting late in life. This result is in agreement with HertzPannier and colleagues' (2002) study. Indeed, they showed that language reorganization occurs in pre-existing bilateral networks which could not be detected preoperatively but postoperatively. This pattern, also called "translocation of language function" (Lazar et al 1997), is interpreted as an over-activity of frontal region with probably taking into account the function of other language regions, such as the posterior (temporo-parietal) ipsilateral areas (Lehericy et al 2002). This pattern could also reflect lower involvement of temporal regions during language, due to their pathological condition (Ries et al 2004).

\section{Parietal region}

Our results indicate an inter-hemispheric reorganization for this region, correlated with the age of seizure onset: higher probability of reorganisation when patients had seizures early in life. These results are in agreement with previous studies which explored the temporo-parietal reorganization for language either by collapsing the two regions (Thivard et al 2005; Helmstaedter et al 2006) or by considering them separately (Hertz-Pannier et al 2002).

\section{Temporal region}

Significant correlation obtained between the signal intensity variation for both, right and left IMTG and the age of seizures onset, suggests that early seizures induced bilateral representation of language for temporal regions. Furthermore, significant correlation of the left IMTG and the age of seizures onset, suggests that the injured hemisphere is still able to deal with language functions (DeVos et al 1995), as the epileptogenic zone is located in the majority of our patients in the left temporal lobe. The correlation obtained between age and the right IMTG is consistent with previous studies showing similar result when the left temporal region is damaged (Hertz-Pannier et al 2002; Brazdil et al 2003; Sabbah et al 2003; Liegeois et al 2004; Thivard et al 2005; Janszky et al 2006).

All together, these results suggest a possible segregation within the left temporal lobe in patients with TLE with seizures starting early in life. Some functional temporal subregions would not be able anymore to take in charge specific language operations. By consequent, their role could be assumed either by other intra-hemispheric language regions (such as frontal; intra-hemispheric "shift") or homologue language regions (right temporal; inter-hemispheric "shift"). Some other functional temporal sub-regions could be overactivated by taking in charge the language functions of those dysfunctional temporal sub-regions.

\section{Associated hippocampal sclerosis}

Hippocampal sclerosis seems playing an important role on the cerebral plasticity for language. In our study, this factor was significantly correlated with the right IMTG activity. Several studies showed right lateralization of the temporal lobe activation in TLE with hippocampal sclerosis (Grunwald et al 
1999; Richardson et al 2003; Schaefer et al 2006; Weber et al 2006). The right "shift" was interpreted either as a compensatory mechanism for verbal memory processes depending on the left damaged hippocampal structures (medial temporal regions) and/or as a compensatory mechanism for language as hippocampal structures could be involved in language lateralization development (Knecht 2004).

Our population of epileptic patients was not homogenous for handedness. The hand preference should be taken into account (Pujol et al 1999; Knecht et al 2000) when studying the language plasticity as linear relationship has been shown between handedness and atypical dominance, with increased incidence of the reorganisation in strong left-handed patients. In the present study, at a first glance, our results did not show correlation between handedness and MR signal intensity values measured in left and right ROIs. This result should be considered very cautiously as the patients sample was very small and the control subjects were not matched with patients for handedness. The influence of the hand preference on language reorganisation will be clearly explored in a greater population of epileptic patients and compared to appropriate and matched left and right-handed healthy subjects in future studies.

\section{Conclusion}

With respect to healthy subjects, the patients with focal TLE explored in the current study show significantly reduced hemispheric lateralization during performing a language task. The significant correlations obtained between the age of seizures onset and the magnitude of temporo-parietal regions suggest supplementary involvement of the right hemisphere (Thivard et al 2005). The tendentious correlation obtained between seizures onset and the magnitude in left frontal region could suggests a possible intra-hemispheric reorganisation of this region, but supplementary examinations should be performed in order to obtain robust significant results. These preliminary results show that cerebral reorganisation could be statistically assessed as a function of different regions and depending on several variables. The sample of patients was small and the results should be considered carefully at the individual level. However, this is encouraging evidence for using neuroimaging methods to study plasticity in further research.

\section{References}

Adcock JE, Wise RG, Oxbury JM, et al. 2003. Quantitative fMRI assessment of the differences in lateralization of language-related brain activation in patients with temporal lobe epilepsy. Neuroimage, 18(2):423-38.

Baciu M, Juphard A, Cousin E, et al. 2005. Evaluating fMRI methods for assessing hemispheric language dominance in healthy subjects. European Journal of Radiology, 55(2):209-18.
Baciu M, Kahane P, Minotti L, et al. 2001. Functional MRI assessment of the hemispheric predominance for language in epileptic patients using a simple rhyme detection task. Epileptic Disorder, 3(3):117-24.

Baciu M, Watson JM, Maccotta L, et al. 2005. Evaluating functional MRI procedures for assessing hemispheric language dominance in neurosurgical patients. Neuroradiology, 47(11):835-44.

Bell B, Hermann B, Seidenberg M, et al. 2002. Ipsilateral Reorganization of Language in Early-Onset Left Temporal Lobe Epilepsy. Epilepsy Behav, 3(2):158-164.

Berl MM, Balsamo LM, Xu B, et al. 2005. Seizure focus affects regional language networks assessed by fMRI. Neurology, 65(10):1604-11.

Billingsley RL, McAndrews MP, Crawley AP, et al. 2001. Functional MRI of phonological and semantic processing in temporal lobe epilepsy. Brain, 124(6):1218-27.

Binder JR, Frost JA, Hammeke TA, et al. 1997. Human brain language areas identified by functional magnetic resonance imaging. J Neurosci, 17(1):353-62.

Binder JR, Swanson SJ, Hammeke TA, et al. 1996. Determination of language dominance using functional MRI: a comparison with the Wada test. Neurology, 46(4):978-84.

Brazdil M, Chlebus P, Mikl M, et al. 2005. Reorganization of languagerelated neuronal networks in patients with left temporal lobe epilepsy an fMRI study. Eur J Neurol, 12(4):268-75.

Brazdil M, Zakopcan J, Kuba R, et al. 2003. Atypical hemispheric language dominance in left temporal lobe epilepsy as a result of the reorganization of language functions. Epilepsy Behav, 4(4):414-9.

Breier JI, Castillo EM, Simos PG, et al. 2005. Atypical language representation in patients with chronic seizure disorder and achievement deficits with magnetoencephalography. Epilepsia, 46(4):540-8.

Corbetta M, Akbudak E, Conturo TE, et al. 1998. A common network of functional areas for attention and eye movements. Neuron, 21(4):761-73.

Cousin E, Peyrin C, Baciu M. 2006. Hemispheric predominance assessment of phonology and semantics: a divided visual field experiment. Brain Cogn, 61(3):298-304.

Demonet JF, Chollet F, Ramsay S, et al. 1992. The anatomy of phonological and semantic processing in normal subjects. Brain, 115(6):1753-68.

Demonet JF, Thierry G, Cardebat D. 2005. Renewal of the neurophysiology of language: functional neuroimaging. Physiol Rev, 85(1):49-95.

DeVos KJ, Wyllie E, Geckler C, et al. 1995. Language dominance in patients with early childhood tumors near left hemisphere language areas. Neurology, 45(2):349-56.

Engel J, Jr. 2001. Mesial temporal lobe epilepsy: what have we learned? Neuroscientist, 7(4):340-52.

Fiez JA. 2001. Neuroimaging studies of speech an overview of techniques and methodological approaches. J Commun Disord, 34(6):445-454.

Friston KJ, Fletcher P, Josephs O, et al. 1998. Event-related fMRI: characterizing differential responses. Neuroimage, 7(1):30-40.

Friston KJ, Frith CD, Frackowiak RS, et al. 1995. Characterizing dynamic brain responses with fMRI: a multivariate approach. Neuroimage, 2(2):166-72.

Gitelman DR, Nobre AC, Sonty S, et al. 2005. Language network specializations: an analysis with parallel task designs and functional magnetic resonance imaging. Neuroimage, 26(4):975-85.

Goldmann RE, Golby AJ. 2005. Atypical language representation in epilepsy: implications for injury-induced reorganization of brain function. Epilepsy Behav, 6(4):473-87.

Grunwald T, Beck H, Lehnertz K, et al. 1999. Limbic P300s in temporal lobe epilepsy with and without Ammon's horn sclerosis. Eur J Neurosci, 11(6):1899-906.

Helmstaedter C, Fritz NE, Gonzalez Perez PA, et al. 2006. Shift-back of right into left hemisphere language dominance after control of epileptic seizures: evidence for epilepsy driven functional cerebral organization. Epilepsy Res, 70(2-3):257-62.

Hertz-Pannier L, Chiron C, Jambaque I, et al. 2002. Late plasticity for language in a child's non-dominant hemisphere: a pre- and post-surgery fMRI study. Brain, 125(Pt 2):361-72. 
Janszky J, Jokeit H, Heinemann D, et al. 2003. Epileptic activity influences the speech organization in medial temporal lobe epilepsy. Brain, 126(Pt 9):2043-51.

Janszky J, Mertens M, Janszky I, et al. 2006. Left-sided interictal epileptic activity induces shift of language lateralization in temporal lobe epilepsy: an fMRI study. Epilepsia, 47(5):921-7.

Kadis DS, Iida K, Kerr EN, et al. 2007. Intrahemispheric reorganization of language in children with medically intractable epilepsy of the left hemisphere. J Int Neuropsychol Soc, 13(3):505-16.

Knecht S. 2004. Does language lateralization depend on the hippocampus? Brain, 127(Pt 6):1217-8.

Knecht S, Drager B, Deppe M, et al. 2000. Handedness and hemispheric language dominance in healthy humans. Brain, 123 (12):2512-8.

Kurthen M, Linke DB, Elger CE, et al. 1992. Linguistic perseveration in dominant-side intracarotid amobarbital tests. Cortex, 28(2):209-19.

Lazar RM, Marshall RS, Pile-Spellman J, et al. 1997. Anterior translocation of language in patients with left cerebral arteriovenous malformation. Neurology, 49(3):802-8.

Lehericy S, Biondi A, Sourour N, et al. 2002. Arteriovenous brain malformations: is functional MR imaging reliable for studying language reorganization in patients? Initial observations. Radiology, 223(3):672-82.

Liegeois F, Connelly A, Cross JH, et al. 2004. Language reorganization in children with early-onset lesions of the left hemisphere: an fMRI study. Brain, 127(Pt 6):1229-36.

Liegeois F, Connelly A, Salmond CH, et al. 2002. A direct test for lateralization of language activation using fMRI: comparison with invasive assessments in children with epilepsy. Neuroimage, 17(4):1861-7.

Lüders HO, Najm I, Nair D, et al. 2006. The epileptogenic zone: general principles. Epileptic Disord, 8 (Suppl 2):1-9.

Lurito JT, Kareken DA, Lowe MJ, et al. 2000. Comparison of rhyming and word generation with FMRI. Hum Brain Mapp, 10(3):99-106.

Maccotta L, Buckner RL, Gilliam FG, et al. 2007. Changing frontal contributions to memory before and after medial temporal lobectomy. Cereb Cortex, 17(2):443-56.

Maldjian J, Laurienti P, Burdette J. 2003. Kraft RAAn Automated Method for Neuroanatomic and Cytoarchitectonic Atlas-based Interrogation of fMRI Data Sets. NeuroImage, 19:1233-9.

Maldjian J, Laurienti P, Burdette J. 2004. Precentral Gyrus Discrepancy in Electronic Versions of the Talairach Atlas. Neuroimage, 21(1):450-5.

Muller RA, Behen ME, Rothermel RD, et al. 1999b. Brain organization for language in children, adolescents, and adults with left hemisphere lesion: a PET study. Prog Neuropsychopharmacol Biol Psychiatry, 23(4):657-68

Muller RA, Rothermel RD, Behen ME, et al. 1999a. Language organization in patients with early and late left-hemisphere lesion: a PET study. Neuropsychologia, 37(5):545-57.

Ojemann G, Ojemann J, Lettich E, et al. 1989. Cortical language localization in left, dominant hemisphere. An electrical stimulation mapping investigation in 117 patients. J Neurosurg, 71(3):316-26.

Oldfield RC. 1971. The assessment and analysis of handedness: the Edinburgh inventory. Neuropsychologia, 9(1):97-113.
Pataraia E, Billingsley-Marshall RL, Castillo EM, et al. 2005. Organization of receptive language-specific cortex before and after left temporal lobectomy. Neurology, 64(3):481-7.

Pataraia E, Simos PG, Castillo EM, et al. 2004. Reorganization of languagespecific cortex in patients with lesions or mesial temporal epilepsy. Neurology, 63(10):1825-32.

Price CJ. 2000. The anatomy of language: contributions from functional neuroimaging. J Anat, 197 (Pt 3):335-9.

Pugh KR, Shaywitz BA, Shaywitz SE, et al. 1996. Cerebral organization of component processes in reading. Brain, 119(4):1221-38.

Pujol J, Deus J, Losilla JM, et al. 1999. Cerebral lateralization of language in normal left-handed people studied by functional MRI. Neurology, 52(5):1038-43.

Richardson MP, Strange BA, Duncan JS, et al. 2003. Preserved verbal memory function in left medial temporal pathology involves reorganisation of function to right medial temporal lobe. Neuroimage, 20 (Suppl 1):S112-9.

Ries ML, Boop FA, Griebel ML, et al. 2004. Functional MRI and Wada determination of language lateralization: a case of crossed dominance. Epilepsia, 45(1):85-9.

Rosenow F, Lüders H. 2001. Presurgical evaluation of epilepsy. Brain, 124(Pt 9):1683-700.

Sabbah P, Chassoux F, Leveque C, et al. 2003. Functional MR imaging in assessment of language dominance in epileptic patients. Neuroimage, 18(2):460-67.

Schaefer M, Heinze HJ, Rotte M. 2006. Verbal memory encoding in patients with left-sided hippocampal sclerosis. Neuroreport, 17(11):1219-23.

Seghier ML, Lazeyras F, Pegna AJ, et al. 2004. Variability of fMRI activation during a phonological and semantic language task in healthy subjects. Hum Brain Mapp, 23(3):140-55.

Springer JA, Binder JR, Hammeke TA, et al. 1999. Language dominance in neurologically normal and epilepsy subjects: a functional MRI study. Brain, 122(11):2033-46.

Talairach J, Tournoux P. 1988. Co-planar stereotaxic atlas of the human brain.3-dimensional proportional system: An approach to cerebral imaging. New York: Thieme.

Thivard L, Hombrouck J, du Montcel ST, et al. 2005. Productive and perceptive language reorganization in temporal lobe epilepsy. Neuroimage, 24(3):841-51.

Thomas P, Genton P. 1992. Epilepsies. Paris: Masson.

Voets NL, Adcock JE, Flitney DE, et al. 2006. Distinct right frontal lobe activation in language processing following left hemisphere injury. Brain, 129(Pt 3):754-66.

Weber B, Wellmer J, Reuber M, et al. 2006. Left hippocampal pathology is associated with atypical language lateralization in patients with focal epilepsy. Brain, 129(Pt 2):346-51.

Yuan W, Szaflarski JP, Schmithorst VJ, et al. 2006. fMRI shows atypical language lateralization in pediatric epilepsy patients. Epilepsia, 47(3):593-600 